\title{
Involvement of an intracellular vesicular transport process in naked-sgRNA-mediated TRUE gene silencing
}

\author{
MASATO TAMURA $^{1}$, MITSUOKI KAWANO ${ }^{1,2}$, MARI SATO $^{1}$ and MASAYUKI NASHIMOTO ${ }^{2}$ \\ ${ }^{1}$ Department of Biochemistry and Molecular Biology, Graduate School of Dental Medicine, \\ Hokkaido University, Sapporo, Hokkaido 060-8586; ${ }^{2}$ Department of Applied Life Sciences, \\ Niigata University of Pharmacy and Applied Life Sciences, Niigata, Niigata 956-8603, Japan
}

Received August 29, 2014; Accepted July 17, 2015

DOI: $10.3892 / \mathrm{mmr} .2015 .4160$

\begin{abstract}
RNase ZL-utilizing efficacious gene silencing (TRUE gene silencing) is an RNA-mediated gene expression control technology with therapeutic potential. Recently, our group demonstrated that a heptamer, mh1 (Bcl-2), targeting human Bcl-2 mRNA, can be taken up by cells without the use of any transfection reagents and can induce the apoptosis of leukemia cells. However, little is known regarding the mechanism of naked small guide (sg)RNA uptake by cultured cells. Therefore, in the present study the effects of various inhibitors on the induction of apoptosis by naked sgRNA treatment were investigated in order to identify the uptake pathway required for sgRNA function in cultured cells. Addition of the endocytosis inhibitors chlorpromazine, nystatin or methyl- $\beta$-cyclodextrin together with naked effective sgRNA was unable to diminish the apoptosis-inducing effects of naked sgRNA or the reduction in target mRNA, suggesting that functional uptake of sgRNA by cells is clathrin-, caveolae- and raft-independent. Next, chloroquine, an inhibitor of lysosome acidification, and brefeldin A, an inhibitor that blocks protein transport from the Golgi apparatus to the endoplasmic reticulum were administered. In the presence of these compounds, the apoptosis-inducing effects of naked sgRNA were reduced. These results suggest that a vesicular transport process is involved in sgRNA-mediated TRUE gene silencing. A greater understanding of how naked sgRNAs enter cells and how they reach their target RNAs may aid in the design of more specifically-targeted and potent sgRNA drugs.
\end{abstract}

Correspondence to: Professor Masato Tamura, Department of Biochemistry and Molecular Biology, Graduate School of Dental Medicine, Hokkaido University, North 13, West 7, Sapporo, Hokkaido 060-8586, Japan

E-mail: mtamura@den.hokudai.ac.jp

Abbreviations: TRUE gene silencing, tRNase ZL-utilizing efficacious gene silencing; sgRNA, small guide RNA; CPZ, chlorpromazine; Nys, nystatin; MbCD, methyl- $\beta$-cyclodextrin

Key words: TRUE gene silencing, small guide RNA, vesicular transport

\section{Introduction}

tRNase ZL-utilizing efficacious gene silencing (TRUE gene silencing) is an RNA-mediated gene expression control technology with therapeutic potential (1-6). This technology is based on a unique enzymatic property of mammalian tRNase ZL, which is that it can cleave any target RNA at a desired site by recognizing a pre-tRNA-like or micro-pre-tRNA-like complex formed between the target RNA and an artificial small guide RNA (sgRNA).

The efficacy of TRUE gene silencing has been demonstrated by using it to introduce various artificially-designed sgRNAs into living cells either by their expression plasmids or by 2'-O-methyl RNAs $(2,7)$. sgRNA is divided into four groups: 5'-half-tRNA, RNA heptamer, hook RNA and $~ 14$-nt linear RNA $(1,2,8,9)$. Linear-type sgRNAs can downregulate the expression of the human genes Bcl-2 and GSK-3 $\beta$ (7). Luciferase gene expression in mouse liver can be inhibited by a heptamer-type sgRNA (7). In addition, the efficacy of TRUE gene silencing can be compared with that of RNA interference technology (6-8). sgRNA can be easily taken up by cultured cells without any transfection reagents, and naked sgRNAs targeting Bcl-2 or WT1 mRNA can reduce the levels of mRNA and protein, as well as inducing the apoptosis of leukemia cells $(9,10)$. However, little is known regarding the mechanism of naked sgRNA uptake into cultured cells. The 'gymnotic' mechanism (11-13) and/or the adaptor protein AP2M1 (14) are reported to be involved in the cellular uptake of naked oligonucleotides by cultured cells. In the present study, the effects of various inhibitors of apoptotic induction were investigated using naked $\operatorname{sgRNA}$ treatment in order to elucidate uptake pathways and mechanisms for sgRNA in cultured cells.

\section{Materials and methods}

RNA/DNA synthesis. The following 5'- and/or 3'-phosphorylated sgRNAs with full 2'-O-methyl modifications were chemically synthesized by Nippon Bioservice (Asaka, Saitama, Japan): mh1 (Bcl-2), 5'-pGGGCCAGp-3'; Luc-hep2, 5'-pGAUCGAG-3'; mh3 (EGFP), 5-pUUGCCGUp-3; Hep5 (HSP90AA1) 5'-pAGAUCCUp-3'; mh2 (FGFR3), 5'-pCCAGCACp-3' (2,9,10). The DNA primers for polymerase chain reaction (PCR) were obtained from Hokkaido System Science (Sapporo, Japan). 




Figure 1. Effects of endocytosis inhibitors on sgRNA-induced apoptosis in HL-60 cells. HL-60 cells were plated at 1x10³ cells/well in a $96-$ well dish in medium (100 $\mu \mathrm{l}$ ) containing $1 \mu \mathrm{M}$ of naked mh1 (Bcl-2) or mh3 (EGFP) (control) with the indicated dose of CPZ, or MbCD. After the cells were cultured for a further 3 days, the numbers of living cells were counted. Each assay represents a separate experiment performed in triplicate. Data are presented as the mean \pm standard deviation. CPZ, chlorpromazine; Nys, nystatin; MbCD, methyl- $\beta$-cyclodextrin.

Cell cultures. HL-60 or HEK-293 human cells were obtained from RIKEN BioResource Center (Tsukuba, Japan) and were cultured in RPMI-1640 medium (Sigma-Aldrich, St. Louis, MO, USA) or $\alpha$-minima essential medium (MEM; Sigma-Aldrich) containing $100 \mu \mathrm{g} / \mathrm{ml}$ kanamycin (Meiji Seika Pharma International Ltd., Tokyo, Japan) and $10 \%$ fetal bovine serum (FBS; SAFC Biosciences, Inc. Lenexa, KS, USA) at $37^{\circ} \mathrm{C}$ in culture flasks or 100-mm culture dishes (Corning, Corning, $\mathrm{NY}, \mathrm{USA}$ ) in a humidified atmosphere of $5 \% \mathrm{CO}_{2}$. The cells were incubated with medium containing chlorpromazine (CPZ), nystatin (Nys), methyl- $\beta$-cyclodextrin (MbCD), heparan sulfate, $10 \mathrm{~K}$ dextran sulfate, chloroquine, brefeldin A or none (vehicle group). The cells were cultured either with $1 \mu \mathrm{M}$ naked 2'-O-methyl RNA or without naked 2'-O-methyl RNA (control cultures).

Compounds and reagents. $\mathrm{CPZ}, \mathrm{Nys}, \mathrm{MbCD}$, heparan sulfate, $10 \mathrm{~K}$ dextran sulfate, chloroquine and brefeldin A were purchased from Sigma-Aldrich.

Quantitation of living cell numbers. HL-60 cells were seeded at a density of $1 \times 10^{3}$ cells/well in a 96-well plate in RPMI-1640 medium and were incubated for $30 \mathrm{~min}$ with medium containing CPZ, Nys, MbCD, heparan sulfate, $10 \mathrm{~K}$ dextran sulfate, chloroquine or brefeldin $\mathrm{A}$, as appropriate, at the concentrations shown. The cells were cultured with $1 \mu \mathrm{M}$ of naked 2'-O-methyl RNA; appropriate vehicle buffer was added to control cultures. After 3 days, to quantitate the number of living cells by the tetrazolium-based colorimetric MTT assay, $20 \mu \mathrm{l}$ TetraColor ONE (Seikagaku Biobusiness Corp., Tokyo, Japan) was added to each well. After $2-4 \mathrm{~h}$ of incubation at $37^{\circ} \mathrm{C}$, the optical density was measured at a wavelength of $450 \mathrm{~nm}$ using a microplate reader (iMark; Bio-Rad Laboratories, Inc., Hercules, CA, USA).

Reverse transcription-PCR (RT-PCR). PCR was used to analyze the transcript levels of hsp90AA1. Total RNA was extracted from the cells at the indicated time points using Isogen (Nippongene, Toyama, Japan) and treated with RNase-free
DNase (Qiagen, Hilden, Germany) to remove any contaminating genomic DNA. RT-PCR was performed as previously described (15). The primer sequences for each gene were as follows: hsp90AA1, 5'-TGCACCTTGGCTCTGTCTGAA-3' (forward), 5'-CACCTGTTAACTGGTACCAAG-3' (reverse); glyceraldehyde-3-phosphate dehydrogenase (GAPDH), 5'-TCCACCACCCTGTTGCTGTA-3' (forward), 5'-ACCACAGTCCATGCCATCAC-3' (reverse). To account for any difference in the quantity of RNA, GAPDH was selected as the endogenous control and amplified using the primers described above. The amplification products were electrophoresed on $2 \%$ agarose gels.

Quantitation of gene expression using reverse transcription-quantitative polymerase chain reaction ( $R T-q P C R)$. RT-qPCR was performed using assay-on-demand TaqMan probes (Applied Biosystems, Foster City, CA, USA) and the StepOne ${ }^{\circledR}$ real time PCR system as previously described (16). The relative level of gene expression was quantified using the comparative $\mathrm{Ct}$ method with GAPDH as the endogenous control.

Statistical analysis. All experiments were repeated at least three times and representative results are shown. In the RT-qPCR analysis and the cell viability assay, differences between control and experimental groups are reported as the mean \pm standard deviation, and were analyzed by Student's $\mathrm{t}$-test, in which values of $\mathrm{P}<0.05$ were considered to indicate a statistically significant difference.

\section{Results and Discussion}

To investigate the properties of heptamer-type sgRNA, our group designed a heptamer, mh1 (Bcl-2), targeting the human Bcl-2 mRNA (9). mh1 (Bcl-2) has the potential to bind Bcl-2 mRNA at four sites, each of which has an immediate upstream sequence that can form a hairpin structure resembling a T-arm of tRNA. As a result, a micro-pre-tRNA-like complex can 
A

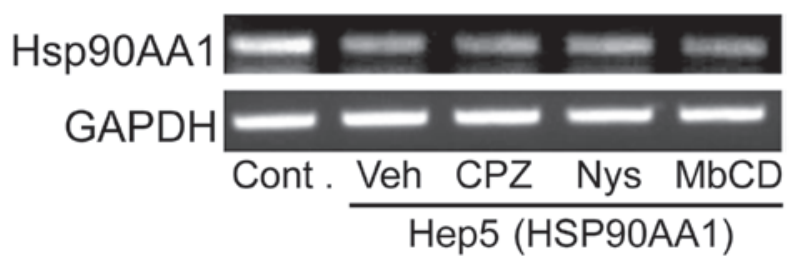



Figure 2. Effects of endocytosis inhibitors on the small guide RNA-induced reduction in hsp90AA1 mRNA in HEK-293 cells. HEK-293 cells were plated at $1 \times 10^{3}$ cells/well in medium $(100 \mu \mathrm{l}$ ) containing $1 \mu \mathrm{M}$ naked Hep5 (HSP90AA1) with CPZ, Nys, MbCD or vehicle. After the cells were cultured for a further 3 days, total RNA was extracted from the cells and the mRNA levels of hsp90AA1 were determined by (A) reverse transcription-PCR or (B) reverse transcription-quantitative PCR. Each assay represents a separate experiment performed in triplicate. Data are presented as the mean \pm standard deviation. Control, without Hep5 (HSP90AA1). "P<0.05 vs. control. CPZ, chlorpromazine; Nys, nystatin; MbCD, methyl- $\beta$-cyclodextrin; PCR, polymerase chain reaction.



Figure 3. Effects of heparan sulfate or dextran sulfate on small guide RNA-induced apoptosis in HL-60 cells. HL-60 cells were plated at 1x10 cells/well in a 96-well plate in medium $(100 \mu \mathrm{l}$ ) containing $1 \mu \mathrm{M}$ naked mh2 (FGFR3) or Luc-hep2 (control) with the indicated dose of heparan sulfate or dextran sulfate. After the cells were cultured for a further 3 days, the numbers of living cells were quantified. Each assay represents a separate experiment performed in triplicate. Data are presented as the mean \pm standard deviation.


Figure 4. Effects of chloroquine or brefeldin A on small guide RNA-induced apoptosis in HL-60 cells. HL-60 cells were plated at 1x10³ cells/well in a 96-well plate in medium (100 $\mu \mathrm{l}$ ) containing $1 \mu \mathrm{M}$ naked mh2 (FGFR3) or mh3 (EGFP) (control) with the indicated dose of (A) chloroquine or (B) brefeldin A. After the cells were cultured for a further 3 days, the numbers of living cells were quantified by the tetrazolium-based colorimetric MTT assay. Each assay represents a separate experiment performed in triplicate. Data are presented as the mean \pm standard deviation. ${ }^{~} \mathrm{P}<0.05$ vs. control. 
be formed at each site, which acts as a substrate for tRNase ZL (2). It was identified that mh1 (Bcl-2) downregulated Bcl-2 mRNA compared with mock or mh3 (EGFP) sgRNA which have no potential target sites on Bcl-2 mRNA. This suggested that naked mh1 (Bcl-2) directs Bcl-2 mRNA cleavage by cellular tRNase ZL (9). First, the effects of fully synthesized 2'-O-methylated mh1 (Bcl-2) containing 5' and 3' phosphates were tested on living cell numbers by adding it to HL-60 cell culture medium without any transfection reagents. When $1 \mu \mathrm{M}$ of naked mh1 (Bcl-2) was added to the culture medium, the living cell number reduced in 3 days compared with cultures without sgRNA (9). By contrast, mh3 (EGFP), that has no potential target site on Bcl-2 mRNA, had no effect on the living cell number (9).

To elucidate the mechanism of naked sgRNA uptake using cultured cells, the effects of various endocytosis inhibitors on sgRNA-induced apoptosis were examined. CPZ disrupts clathrin-dependent endocytosis, while Nys selectively disrupts caveolae- and lipid raft-dependent endocytosis but has no effect on clathrin-dependent endocytosis. MbCD disrupts both lipid raft- and clathrin-dependent endocytosis (17). HL-60 cells were incubated for $30 \mathrm{~min}$ without (control) or with the different inhibitors, and then incubated with $1 \mu \mathrm{M}$ of mh1 (Bcl-2) for 3 days. Addition of any concentration of the compounds in combination with naked effective sgRNA was unable to diminish its effect on apoptosis in HL-60 cells (Fig. 1), indicating that sgRNA-induced apoptosis does not depend upon caveolae-, lipid raft- or clathrin-dependent endocytic mechanisms in these cells.

The effects of various endocytosis inhibitors on sgRNA-directed mRNA reduction in HEK-293 cells were examined. Treatment with Hep5 (HSP90AA1) resulted in a reduction of hsp90AA1 mRNA levels in HEK-293 cells (Fig. 2). None of the endocytosis inhibitors CPZ, NYS or MbCD had any inhibitory effect on the action of sgRNA in reducing hsp90AA1 mRNA as compared with the vehicle (Fig. 2). These observations also suggested that functional uptake of sgRNA by cells is clathrin-, caveolae- and lipid raft-independent.

To determine the ability of polyanions to compete for functional cellular sgRNA uptake and sgRNA-induced apoptosis, heparan sulfate and dextran sulfate were used. Neither heparan sulfate nor dextran sulfate were able to attenuate the effect of mh2 (FGFR3) on apoptosis in HL-60 cells (Fig. 3). These results demonstrate that sgRNA activities do not only occur via interactions of polyanions with membrane proteins, but appear to be also due to recognition of both base and sugar.

Next, two pharmacological inhibitors of endocytic processes were used to further define the pathways through which sgRNA could induce apoptosis in the functional compartment of cells. Chloroquine, an inhibitor of lysosome acidification, and brefeldin A (BFA), a lactone antibiotic that blocks retrograde protein transport from the Golgi apparatus to the endoplasmic reticulum (18) were administered. In the presence of these compounds, the induction of apoptosis by naked effective mh2 (FGFR3) was reduced (Fig. 4). These results suggest that the uptake of functional sgRNA into cells involves a vesicular transport process.

Chemically modified single-stranded oligonucleotides, delivered systemically, rapidly disperse out of the plasma and are taken up by cells within tissues (19-21). Although transfection agents are required to produce effects of single-stranded oligonucleotides in the majority of cultured cells, several investigations have indicated that single-stranded oligonucleotides can be taken up and exert their effects without any transfection agent (11). It was also demonstrated that a heptamer, mh1 (Bcl-2), which targets human Bcl-2 mRNA, can be taken up by cells without any transfection reagents and that it can induce apoptosis of leukemia cells (9). However, it is not fully understood how polyanionic oligonucleotides gain access to the cytoplasmic and/or nuclear compartments of the cell where they can bind to the target RNA.

Endocytosis describes the de novo production of internal membranes from the plasma membrane lipid bilayer (22). In clathrin-mediated endocytosis, proteins involved in this process recruit cargo into developing clathrin-coated pits, and subsequently form clathrin-coated vesicles. By contrast, clathrin-independent endocytosis is hypothesized to occur by seemingly distinct pathways, based on the reliance of these pathways on certain proteins and lipids. In the present study, none of the endocytosis inhibitors CPZ, Nys or MbCD were able to diminish the effects of apoptosis induction or target mRNA reduction by sgRNA. These results indicate that functional uptake by cells in culture does not appear to be mediated by clathrin-, caveolae- or lipid raft-dependent pathways. Similarly to our studies, Koller et al (14) reported that functional uptake of a single-stranded phosphorothioate modified antisense oligonucleotide is not mediated by clathrin or caveolin.

Following cellular uptake, oligonucleotides are sequestered in intracellular compartments of unknown nature, from which they are hypothesized to escape by an undetermined and presumably inefficient mechanism. Based on relief by monensin of fluorescence quenching, fluoresceinated phosphorothioate oligonucleotides were reported to be identified in acidic compartments, compatible with lysosomes (23). The present study suggested that functional uptake of sgRNA is an intracellular vesicular uptake process, since it is blocked by choroquine and brefeldin A. Recently, it has been reported that inhibition of the adaptor protein AP2M1 with small interfering RNA attenuated the antisense effects in cultured hepatocytes $(14,24)$. Additional studies are required to determine whether AP2M1 mediates the effects of naked sgRNAs and to fully identify methods of sgRNA uptake. A greater understanding of how naked sgRNAs enter cells and how they reach their target RNA may aid in the design of more specifically-targeted and more potent sgRNA drugs.

\section{Acknowledgements}

This study was supported by the Adaptable and Seamless Technology Transfer Program through Target-driven R\&D, Japan Science and Technology Agency (to Professor Masayuki Nashimoto).

\section{References}

1. Nashimoto M: Specific cleavage of target RNAs from HIV-1 with 5' half tRNA by mammalian tRNA 3' processing endoribonuclease. RNA 2: 523-524, 1996. 
2. Tamura M, Nashimoto C, Miyake N, Daikuhara Y, Ochi K and Nashimoto M: Intracellular mRNA cleavage by 3 ' tRNase under the direction of 2'-O-methyl RNA heptamers. Nucleic Acids Res 31: 4354-4360, 2003.

3. Nashimoto M: Anomalous RNA substrates for mammalian tRNA 3' processing endoribonuclease. FEBS Lett 472: 179-186, 2000.

4. Takaku H, Minagawa A, Takagi M and Nashimoto M: A novel 4-base-recognizing RNA cutter that can remove the single 3' terminal nucleotides from RNA molecules. Nucleic Acids Res 32: e91, 2004

5. Shibata HS, Takaku H, Takagi M and Nashimoto M: The T loop structure is dispensable for substrate recognition by tRNase ZL. J Biol Chem 280: 22326-22334, 2005.

6. Elbarbary R, Takaku H, Tamura $M$ and Nashimoto $M$ : Inhibition of vascular endothelial growth factor expression by TRUE gene silencing. Biochem Biophys Res Commun 379: 924-927, 2009.

7. Nakashima A, Takaku H, Shibata HS, Negishi Y, Takagi M, Tamura $M$ and Nashimoto $M$ : Gene silencing by the tRNA maturase tRNase $\mathrm{Z}(\mathrm{L})$ under the direction of small-guide RNA. Gene Ther 14: 78-85, 2007.

8. Sano T, Takahashi M, Nozaki T, Takahashi Y, Tamura M and Nashimoto M: Expanding the utility of heptamer-type sgRNA for TRUE gene silencing. Biochem Biophys Res Commun 416: 427-432, 2011

9. Takahashi M,Elbarbary RA, Nakashima A, Abe M, Watanabe N, Narita M, Takahashi M, Tamura M, Yoshida T and Nashimoto M: A naked RNA heptamer targeting the human Bcl-2 mRNA induces apoptosis of HL60 leukemia cells. Cancer Letters 328 362-368, 2013

10. Watanabe N, Narita M, Yamahira A, Taniguchi T, Furukawa T, Yoshida T, Miyazawa T, Nashimoto $M$ and Takahashi $M$ : Induction of apoptosis of leukemic cells by TRUE gene silencing using small guide RNAs targeting the WT1 mRNA. Leuk Res 37: 580-585, 2013.

11. Stein CA, Hansen JB, Lai J, Wu S, Voskresenskiy A, Høg A, Worm J, Hedtjärn M, Souleimanian N, Miller P, et al: Efficient gene silencing by delivery of locked nucleic acid antisense oligonucleotides, unassisted by transfection reagents. Nucleic Acids Res 38: e3, 2010.

12. Soifer HS, Souleimanian N, Wu S, Voskresenskiy AM, Collak FK, Cinar B and Stein CA: Direct regulation of androgen receptor activity by potent CYP17 inhibitors in prostate cancer cells. J Biol Chem 287: 3777-3787, 2012.
13. Souleimanian N, Deleavey GF, Soifer H, Wang S, Tiemann K, Damha MJ and Stein CA: Antisense 2'-deoxy, 2'-fluroarabino nucleic acids (2'F-ANAs) oligonucleotides: In vitro gymnotic silencers of gene expression whose potency is enhanced by fatty acids. Mol Ther Nucleic Acids 1: e43, 2012.

14. Koller E, Vincent TM, Chappell A, De S, Manoharan M and Bennett CF: Mechanisms of single-stranded phosphorothioate modified antisense oligonucleotide accumulation in hepatocytes. Nucleic Acids Res 39: 4795-4807, 2011

15. Sato MM, Nakashima A, Nashimoto M, Yawaka Y and Tamura M: Bone morphogenetic protein-2 enhances Wnt/beta-catenin signaling-induced osteoprotegerin expression. Genes Cells 14: $141-153,2009$.

16. Uyama M, Sato MM, Kawanami M and Tamura M: Regulation of osteoblastic differentiation by the proteasome inhibitor bortezomib. Genes Cells 17: 548-558, 2012.

17. Dutta D and Donaldson JG: Search for inhibitors of endocytosis: Intended specificity and unintended consequences. Cell Logist 2: 203-208, 2012.

18. Donaldson JG, Finazzi D and Klausner RD: Brefeldin A inhibits Golgi membrane-catalysed exchange of guanine nucleotide onto ARF protein. Nature 360: 350-352, 1992.

19. Kastelein JJ, Wedel MK, Baker BF, Su J, Bradley JD, Yu RZ, Chuang E, Graham MJ and Crooke RM: Potent reduction of apolipoprotein B and low-density lipoprotein cholesterol by short-term administration of an antisense inhibitor of apolipoprotein B. Circulation 114: 1729-1735, 2006.

20. Sazani P, Gemignani F, Kang SH, Maier MA, Manoharan M, Persmark M, Bortner D and Kole R: Systemically delivered antisense oligomers upregulate gene expression in mouse tissues. Nat Biotechnol 20: 1228-1233, 2002.

21. Vickers TA, Zhang H, Graham MJ, Lemonidis KM, Zhao C and Dean NM: Modification of MyD88 mRNA splicing and inhibition of IL-1beta signaling in cell culture and in mice with a 2'-O-methoxyethyl-modified oligonucleotide. J Immunol 176 : 3652-3661, 2006.

22. Doherty GJ and McMahon HT: Mechanisms of endocytosis. Annu Rev Biochem 78: 857-902, 2009.

23. Tonkinson JL and Stein CA: Patterns of intracellular compartmentalization, trafficking and acidification of 5'-fluorescein labeled phosphodiester and phosphorothioate oligodeoxynucleotides in HL60 cells. Nucleic Acids Res 22: 4268-4275, 1994.

24. Bennett CF and Swayze EE: RNA targeting therapeutics: Molecular mechanisms of antisense oligonucleotides as a therapeutic platform. Annu Rev Pharmacol Toxicol 50: 259-293, 2010. 